\title{
Multimorbidity in Chronic Conditions: Public Primary Care Patients in Four Greater Mekong Countries
}

\author{
Supa Pengpid ${ }^{1,2}$ and Karl Peltzer ${ }^{2,3, *}$ \\ 1 ASEAN Institute for Health Development, Mahidol University, Salaya, Phutthamonthon, \\ Nakhon Pathom 73170, Thailand; supaprom@yahoo.com \\ 2 Department of Research Innovation and Development, University of Limpopo, Sovenga 0727, South Africa \\ 3 HIV/AIDS/STIs and TB (HAST), Human Sciences Research Council, Pretoria 0001, South Africa \\ * Correspondence: kpeltzer@hsrc.ac.za; Tel.: +27-12-302-2000; Fax: +27-12-302-2067 \\ Academic Editor: Noël Christopher Barengo \\ Received: 19 August 2017; Accepted: 5 September 2017; Published: 6 September 2017
}

\begin{abstract}
The aim of this study was to explore the prevalence, pattern, and social determinants of chronic conditions multimorbidity among chronic disease primary care patients in four Greater Mekong countries (Cambodia, Myanmar, Thailand, and Vietnam). In a cross-sectional survey, chronic disease patients accessing primary care were recruited if they had been diagnosed with any of 21 chronic conditions in the past 12 months, and were interviewed with a structured questionnaire on anxiety, depression, alcohol use, tobacco use, dietary behaviour, physical activity, and quality of life. The sample included 6236 public primary care patients $(32.8 \%$ men and $67.2 \%$ women), with a mean age of 53.0 years $(\mathrm{SD}=16.8)$. From 21 chronic conditions, the three most common were hypertension (37.4\%), depression (34.4\%), and digestive diseases (32.0\%). In all, $27.4 \%$ had one chronic condition, $28.6 \%$ had two, $22.4 \%$ had three, and $21.6 \%$ had four or more chronic conditions. The percentage with the highest comorbidity was depression $(47.3 \%)$, hypertension $(43.4 \%)$, and digestive diseases $(34.1 \%)$. The highest mean multimorbidity reported was for mental illness (4.44), kidney disease (4.11), and Parkinson's disease (4.10), and the lowest multimorbidity for epilepsy (2.43) and cancer (2.80). Compared to those who had only one chronic condition, being male, older age, lower education, and lower quality of life were associated with having two and three or more chronic conditions. Multimorbidity is a prevalent problem among chronic condition primary care patients-a finding with implications for health care delivery, management, and research.
\end{abstract}

Keywords: chronic condition; multimorbidity; public primary care; Greater Mekong countries

\section{Introduction}

There has been a rapid increase in non-communicable disease (NCD)-related deaths in Asian—including Greater Mekong—countries. NCDs—in particular cardiovascular disorders (CVDs), cancer, diabetes, and chronic respiratory disease-are now the major cause of death and disease burden in the region [1,2]. The epidemiological transition from communicable to NCDs has been taking place in Southeast Asia, creating a public health challenge [3]. NCDs are the number-one killer in the South Asia region, accounting for 55\% of all death [4]. Globally, half of the people with NCDs have two or more conditions (multimorbidity) [5]. Multimorbidity may be defined as "the coexistence of multiple chronic conditions in a given individual" [6]. Based on the research to date [5], there seems to be no consensus on which conditions should be considered or on the method used for measuring multimorbidity [7]. Evidence from low-income settings is limited [7-9]. 
In a South African primary health care study, hypertension was the most common comorbid condition in type 2 diabetes, epilepsy, asthma, and chronic obstructive pulmonary disease (COPD), and diabetes and hypertension was the most common combination [10]. Chronic condition (hypertension, diabetes, asthma, COPD, epilepsy, and osteoarthritis) multimorbidity (two or more conditions) was present in $36.4 \%$ of patients with COPD, $23.7 \%$ with osteoarthritis, $16.3 \%$ with diabetes, $15.3 \%$ with asthma, $12.0 \%$ with hypertension, and $6.7 \%$ with epilepsy [10]. About half of the patients with chronic conditions had comorbidity, and multimorbidity was common in patients with COPD and osteoarthritis [10]. In a large primary care population with one of 24 chronic diseases aged 55 years and older in the Netherlands, the mean multimorbidity was the highest for CVD (3.39-4.97), COPD (3.40), visual disorder (3.38), rheumatoid arthritis (3.28), asthma (3.24), Parkinson's disease (3.23), anxiety disorder (3.21), depression (3.10), osteoarthritis (3.03), and the lowest in hypertension (2.57), migraine (2.62), cancer (2.85), musculoskeletal disorder (2.95), and epilepsy (2.98) [11]. In a large primary care practice database in the United States, the prevalence of multimorbidity (two or more chronic conditions) of 24 chronic diseases was $45.2 \%$ [12]. A pattern of chronic conditions that was observed was, for example, between atherosclerosis and hypertension, coronary heart disease, diabetes mellitus, and hyperlipidaemia [12].

Factors associated with the multimorbidity of chronic conditions (two or more chronic conditions) may include older age $[8,10,13-15]$, women $[8,13,15]$, better education $[14,15]$, higher wealth index [14,15], lower socioeconomic status [8], urban residence [14], and low quality of life [16]. There is a lack of studies investigating multimorbidity among chronic disease patients, especially in Southeast Asia. Therefore, the aim of this study was to explore the prevalence, pattern, and social determinants of chronic conditions multimorbidity among chronic disease primary care patients in four Greater Mekong countries.

\section{Materials and Methods}

\section{Sample and Procedure}

In the study countries (Cambodia, Myanmar, Thailand, and Vietnam), a cross-sectional survey in rural and urban primary health facilities was conducted among out-patients with chronic diseases. The study facilities were convenience-selected. Each eligible patient aged $\geq 18$ years with a chronic disease was selected from the health facility, using a systematic sampling procedure; more details can be found in $[17,18]$.

Trained research assistants conducted interviews with the patients using structured questionnaires after obtaining informed consent. For this study, patients with one of these 21 chronic conditions were invited in the study: asthma, COPD, diabetes mellitus, hypertension, dyslipidaemia, coronary artery disease, cardiac failure, cardiac arrhythmias, stroke, arthritis, cancer, gout, Parkinson's disease, liver disease, kidney disease, thyroid disease, stomach and intestinal diseases, epilepsy, and mental disorders [11,19]. In addition, information on four chronic conditions (daily tobacco use, alcohol use disorder, anxiety, and depression) and on dietary behaviour, physical activity, and quality of life was assessed with structured questionnaires. In Cambodia, the National Ethics Committee for Health Research, Ministry of Health, Cambodia (Reference No.: 0225NECHR); in Myanmar the Research and Ethical Committee, University of Medicine 1, Yangon; and in Vietnam the Committee for Research Ethics, Hanoi School of Public Health; and in Thailand, the Committee for Research Ethics in Social Sciences, Mahidol University (COA. No.: 2014/193.0807), approved the study protocol.

\section{Measures}

Tobacco use was assessed with four questions: "(1) Do you currently smoke any tobacco products, such as cigarettes, cigars or pipes?" If they answered yes, they were asked "(2) Do you currently smoke tobacco products daily? (3) Do you currently use any smokeless tobacco, such as snuff, chewing 
tobacco, betel?" If they answered yes, they were asked "(4) Do you currently use smokeless tobacco products daily?" [20].

Alcohol use disorder was assessed using the Alcohol Use Disorder Identification Test (AUDIT)-C with a cut-off score of four defined as problem drinking [21] (Cronbach alpha 0.81).

The Hospital Anxiety and Depression Scale (HADS) [22] was used to assess anxiety (seven items) and depression (seven items). A score of $\geq 11$ was used to define anxiety and depression [22] (Cronbach alpha was 0.73 and 0.71 for anxiety and for depression, respectively).

Dietary behaviour was assessed with seven items: consumption of fast food, fruits and vegetables, sweet drinks, protein, chips and crackers, desserts and fats during the previous 7 days [23]. From these seven items, we calculated a total dietary score of up to 14 points with 0 points signifying the best dietary habits and 14 signifying the poorest dietary habits [23].

Physical activity was assessed using the General Physical Activity Questionnaire (GPAQ) [24]. The total physical activity score was used to divide participants into inactive and moderately or highly active [24].

Quality of life was assessed with the World Health Organization Quality of Life (WHOQol)-8, consisting of eight items that were derived from the WHOQOL-Bref [25]. The eight-item index consists of two items from each domain of the original WHOQOL-BREF (physical, psychological, environmental, and social) [26], and showed acceptable cross-cultural performance and a satisfactory discriminant validity [26]. Results from the two-item sub-scales and the eight-item index were summed to get sub-scale and overall WHOQoL scores, which was then transformed to a $0-100$ scale, which was grouped into low, medium, and high QoL (Cronbach alpha 0.88).

Sociodemographic data obtained from all participants were age, sex, country, formal education level, marital status, and residency status.

\section{Data Analysis}

Data were analysed using the IBM Statistical Package for the Social Sciences (SPSS) for Windows version 24.0 (SPSS, Inc., Chicago, IL, USA). The proportions of chronic diseases were calculated as percentages, means, and standard deviations. Multinomial logistic regression was used by comparing two chronic conditions and three or more chronic conditions multimorbidity prevalence with having only one chronic condition (reference category). Predictor variables included sociodemographic factors, quality of life, and behavioural risk factors (physical inactivity and poor diet). Probability below 0.05 was regarded as statistically significant.

\section{Results}

The total sample included 6236 public primary care patients; $67.2 \%$ were female, 1594 from Cambodia, 1533 from Myanmar, 1549 from Thailand, and 1560 from Vietnam. The mean age of the study population was 53.0 years $(\mathrm{SD}=16.8)$, ranging 18 to 94 years. More than half $(59.8 \%)$ were 50 years and older, $62.0 \%$ had six or more years of education, and $45.9 \%$ were residing in urban areas. Almost half of the participants (43.1\%) reported a low quality of life, $29.4 \%$ were physically inactive, and $39.3 \%$ had a poor dietary behaviour.

From 17 health record-assessed chronic conditions (coronary artery disease, cardiac failure, cardiac arrhythmias and stroke were combined into CVD) and four interview-based assessed chronic conditions (depression, anxiety, alcohol use disorder, and daily tobacco use), the most common was hypertension $(37.4 \%)$, followed by depression $(34.4 \%)$, digestive diseases $(32.0 \%)$, anxiety $(19.4 \%)$, musculoskeletal diseases (16.4\%), type 2 diabetes (17.2\%), daily tobacco use $(16.0 \%)$, CVD $(15.9 \%)$, and arthritis (15.9\%). In all, $27.4 \%$ had one chronic condition, $28.6 \%$ had two, $22.4 \%$ had three, and $21.6 \%$ had four or more chronic conditions. The percentage with the highest comorbidity was depression (47.3\%), followed by hypertension (43.4\%), digestive diseases $(34.1 \%)$, anxiety $(26.6 \%)$, daily tobacco use $(22.0 \%)$, CVD $(20.0 \%)$, type 2 diabetes $(18.9 \%)$, arthritis $(18.3 \%)$, and musculoskeletal diseases $(18.1 \%)$. The highest mean multimorbidity reported was for mental illness (4.44), followed by 
kidney disease (4.11), Parkinson's disease (4.10), anxiety (3.74), daily tobacco use (3.62), migraine (3.62), depression (3.58), CVD (3.57), and COPD (3.56), and the lowest multimorbidity was found for epilepsy (2.43), followed by cancer (2.80) and asthma (2.82). Comparing the younger (18-49 years) with the older age group (50-94 years), in most chronic conditions the mean multimorbidity was higher among the older than younger age group, except for a few conditions: CVD, COPD, mental illness, cancer, and Parkinson's disease (see Table 1).

Table 1. Chronic condition morbidity pattern in the sample population.

\begin{tabular}{|c|c|c|c|c|c|}
\hline Chronic Condition & $\%$ & $\begin{array}{l}\text { Percentage } \\
\text { with } \\
\text { Comorbidity }\end{array}$ & $\begin{array}{c}\text { Mean } \\
\text { Multimorbidity } \\
\text { (SD)-Total }\end{array}$ & $\begin{array}{c}\text { Mean } \\
\text { Multimorbidity } \\
\text { (SD)-18-49 Years }\end{array}$ & $\begin{array}{c}\text { Mean } \\
\text { Multimorbidity } \\
\text { (SD)-50-94 Years }\end{array}$ \\
\hline Depression & 34.4 & 47.3 & $3.58(1.3)$ & $3.39(1.3)$ & $3.70(1.3)$ \\
\hline Digestive diseases & 32.0 & 34.1 & $2.99(1.7)$ & $2.59(1.6)$ & $3.59(1.7)$ \\
\hline Anxiety & 19.4 & 26.6 & $3.74(1.3)$ & $3.51(1.3)$ & $3.88(1.3)$ \\
\hline Daily tobacco use & 16.0 & 22.0 & $3.62(1.4)$ & $3.45(1.4)$ & $3.70(1.7)$ \\
\hline $\mathrm{CVD}^{1}$ & 15.9 & 20.0 & $3.57(1.7)$ & $3.83(1.9)$ & $3.47(1.6)$ \\
\hline Arthritis & 15.9 & 18.3 & $3.54(1.8)$ & $3.26(1.9)$ & $3.30(1.6)$ \\
\hline Dyslipidaemia & 10.4 & 13.8 & $3.46(1.4)$ & $3.06(1.4)$ & $3.64(1.4)$ \\
\hline Alcohol use disorder & 7.8 & 10.6 & $3.44(1.3)$ & $3.28(1.3)$ & $3.73(1.4)$ \\
\hline Migraine or frequent headaches & 7.0 & 8.0 & $3.62(2.0)$ & $3.33(1.9)$ & $4.10(1.8)$ \\
\hline Mental illness & 2.4 & 3.0 & $4.44(2.2)$ & $4.55(2.3)$ & $4.32(2.0)$ \\
\hline Thyroid disease & 1.8 & 2.0 & $3.03(1.8)$ & $2.59(1.6)$ & $3.38(1.7)$ \\
\hline Cancer & 1.5 & 1.6 & $2.80(1.6)$ & $2.93(1.7)$ & $2.74(1.5)$ \\
\hline Parkinson's disease & 1.6 & 1.8 & $4.10(2.6)$ & $5.30(2.6)$ & $3.45(2.3)$ \\
\hline Epilepsy & 0.4 & 0.3 & $2.43(1.9)$ & $2.40(2.0)$ & $2.67(0.6)$ \\
\hline
\end{tabular}

${ }^{1}$ Coronary artery diseases $(186,3.8 \%)$, cardiac failure $(370,7.5 \%)$, cardiac arrhythmias $(144,2.9 \%)$, stroke $(182,3.7 \%)$.

CVD: cardiovascular disease.

Table 2 describes the top ten chronic conditions that were comorbid with six major chronic conditions. Of those who had hypertension, the highest comorbidity was with depression $(34.6 \%)$, type 2 diabetes (24.8\%), CVD (20.6\%), and dyslipidaemia (19.0\%). Hypertension had the highest comorbidity with type 2 diabetes (56.7\%), CVD (48.5\%), and musculoskeletal diseases $(29.4 \%)$. The major comorbid chronic conditions with COPD were depression (56.9\%), digestive diseases $(36.2 \%)$, and daily tobacco use (31.1\%) (see Table 2). 
Table 2. The top ten chronic conditions that were comorbid with each chronic condition. COPD: chronic obstructive pulmonary disease.

\begin{tabular}{|c|c|c|c|c|c|c|c|c|c|c|c|}
\hline \multicolumn{2}{|c|}{ Hypertension $N=2331$} & \multicolumn{2}{|c|}{ Type 2 Diabetes $N=1022$} & \multicolumn{2}{|l|}{ CVD $N=992$} & \multicolumn{2}{|l|}{ COPD $N=351$} & \multicolumn{2}{|c|}{ Arthritis $N=991$} & \multicolumn{2}{|c|}{$\begin{array}{l}\text { Musculoskeletal Diseases } \\
\qquad N=1025\end{array}$} \\
\hline Comorbid Condition & $\%$ & & $\%$ & & $\%$ & & $\%$ & & $\%$ & & $\%$ \\
\hline Depression & 34.6 & Hypertension & 56.7 & Hypertension & 48.5 & Depression & 56.9 & Depression & 47.5 & Hypertension & 29.4 \\
\hline Type 2 diabetes & 24.8 & Depression & 34.3 & Depression & 47.6 & Digestive diseases & 36.2 & Digestive diseases & 40.0 & Digestive diseases & 26.0 \\
\hline CVD & 20.6 & Anxiety & 20.7 & Digestive diseases & 26.2 & Daily tobacco use & 31.1 & Hypertension & 28.5 & Depression & 24.4 \\
\hline Dyslipidaemia & 19.0 & Dyslipidaemia & 19.5 & Anxiety & 23.2 & CVD & 20.8 & $\begin{array}{l}\text { Musculoskeletal } \\
\text { diseases }\end{array}$ & 22.0 & Arthritis & 21.3 \\
\hline Anxiety & 18.9 & Daily tobacco use & 13.1 & Arthritis & 18.4 & Anxiety & 20.6 & Anxiety & 20.8 & Daily tobacco use & 17.1 \\
\hline Daily tobacco use & 16.9 & CVD & 11.3 & Daily tobacco use & 17.9 & Hypertension & 19.4 & CVD & 18.5 & Anxiety & 16.5 \\
\hline Digestive diseases & 14.6 & Digestive diseases & 12.1 & $\begin{array}{l}\text { Musculoskeletal } \\
\text { diseases }\end{array}$ & 13.3 & Arthritis & 17.9 & Daily tobacco use & 18.4 & CVD & 12.9 \\
\hline $\begin{array}{l}\text { Musculoskeletal } \\
\text { diseases }\end{array}$ & 12.9 & Arthritis & 7.5 & Dyslipidaemia & 12.2 & $\begin{array}{l}\text { Musculoskeletal } \\
\text { diseases }\end{array}$ & 12.3 & Migraine & 12.2 & Alcohol use disorder & 10.0 \\
\hline Arthritis & 12.1 & $\begin{array}{l}\text { Musculoskeletal } \\
\text { diseases }\end{array}$ & 7.5 & Type 2 diabetes & 11.6 & Migraine & 8.3 & Kidney disease & 8.4 & Dyslipidaemia & 9.5 \\
\hline Alcohol use disorder & 5.3 & Alcohol use disorder & 3.8 & Migraine & 9.1 & Asthma & 7.4 & Type 2 diabetes & 7.8 & Migraine & 9.4 \\
\hline
\end{tabular}


Compared to those who had only one chronic condition, being male, older age, lower education, and lower quality of life were associated with having two and three or more chronic conditions. In addition, rural residence and poor dietary behaviour were associated with three or more chronic conditions (see Table 3).

Table 3. Multinomial logistic regression model examining correlates of multimorbidity.

\begin{tabular}{|c|c|c|}
\hline Variable & $\begin{array}{l}\text { Two Chronic Conditions versus } \\
\text { One Chronic Condition }\end{array}$ & $\begin{array}{c}\text { Three or More Chronic Conditions } \\
\text { versus One Chronic Condition }\end{array}$ \\
\hline & AOR $(95 \%$ CI $)$ & AOR $(95 \% \mathrm{CI})$ \\
\hline \multicolumn{3}{|l|}{ Sex } \\
\hline Female & 1 (Reference) & 1 (Reference) \\
\hline Male & $1.80(1.43,2.26)^{* * *}$ & $2.19(1.77,2.71)^{* * *}$ \\
\hline \multicolumn{3}{|l|}{ Age (in years) } \\
\hline $18-49$ & 1 (Reference) & 1 (Reference) \\
\hline $50-94$ & $1.36(1.11,1.67) * *$ & $1.83(1.52,2.21)^{* * *}$ \\
\hline \multicolumn{3}{|l|}{ Education } \\
\hline Grade 0-5 & 1 (Reference) & 1 (Reference) \\
\hline Grade 6-11 & $0.78(0.62,0.99) *$ & $0.44(0.36,0.54)^{* * *}$ \\
\hline Grade 12 or more & $0.59(0.44,0.78)^{* * *}$ & $0.30(0.23,0.39)^{* * *}$ \\
\hline \multicolumn{3}{|l|}{ Income } \\
\hline Low & 1 (Reference) & 1 (Reference) \\
\hline High & $1.05(0.85,1.30)$ & $0.97(0.80,1.18)$ \\
\hline \multicolumn{3}{|l|}{ Geolocality } \\
\hline Urban & 1 (Reference) & 1 (Reference) \\
\hline Rural & $1.06(0.86,1.32)$ & $1.24(1.02,1.51)^{*}$ \\
\hline \multicolumn{3}{|l|}{ Quality of Life } \\
\hline Low & 1 (Reference) & 1 (Reference) \\
\hline Medium & $0.69(0.50,0.96)$ * & $0.47(0.34,0.64)^{* * *}$ \\
\hline High & $0.77(0.62,0.96)$ * & $0.65(0.54,0.79)^{* * *}$ \\
\hline \multicolumn{3}{|l|}{ Physical inactivity } \\
\hline No & 1 (Reference) & 1 (Reference) \\
\hline Yes & $0.90(0.73,1.13)$ & $1.20(0.98,1.46)$ \\
\hline Poor diet index (scale) & $1.07(0.88,1.31)$ & $1.26(1.05,1.52) *$ \\
\hline
\end{tabular}

\section{Discussion}

The study found that among chronic condition primary care patients in four Greater Mekong countries, the majority $(72.6 \%)$ had multimorbidity (as also found in previous studies $[5,11,12]$ ), and even higher than in South Africa [10]. This study found that hypertension was the most common comorbid condition in type 2 diabetes, COPD, CVD, and musculoskeletal diseases, while a previous study in South Africa found that it was the most common comorbid condition in type 2 diabetes, epilepsy, asthma, and COPD [10]. Similar to a previous study among primary care patients in the Netherlands with 24 chronic conditions [11], this study found that the highest mean multimorbidity was among patients with mental disorders and CVD and lowest for cancer, epilepsy, and hypertension patients.

As reviewed previously [27-29], in this study depression was a very common comorbid condition of type 2 diabetes, CVD, COPD, and arthritis, and anxiety was a common comorbid condition in CVD. This finding has also been confirmed in a systematic review [30] where "depression was found to be the disease that was most commonly clustered, and was paired with 8 different diseases." Further, as also found in the systematic review [30], this study found that hypertension was the second most clustered disease with type 2 diabetes, CVD, and musculoskeletal diseases, while this was only partially true for 
type 2 diabetes which only highly clustered with hypertension. A common chronic conditions pattern included hypertension, type 2 diabetes, CVD, and dyslipidaemia, which was similarly observed among US American primary care patients [12]. Some of the most common chronic condition comorbidity patterns may be explained by having a common pathophysiology [31].

In agreement with previous studies $[8,10,13-15]$, this study found that older chronic condition patients were more likely to have chronic condition multimorbidity than younger patients, which is consistent with the idea that additional life years add additional opportunities to develop other chronic conditions [8]. Contrary to some previous studies $[8,13,15]$, this study found an association between male gender and chronic condition multimorbidity. The higher prevalence of chronic condition multimorbidity among men in this sample may be because alcohol and tobacco use disorders were included in the 21 chronic conditions assessed. These two substance use disorders were much more common in men than women in this study (analysis not shown). In this study, the likelihood of chronic condition multimorbidity was higher in those with lower education. This finding was also found in some other studies $[8,13]$, while in a study in India and Indonesia $[14,15]$, better education was associated with chronic condition multimorbidity. This study did not find an association between income or economic status and chronic condition multimorbidity, while other studies found mixed results $[8,14,15]$.

This study may confirm the existence of an inverse association between chronic condition multimorbidity and quality of life, as found in a previous review [16]. The possible association between chronic condition multimorbidity and quality of life may have additional health care management implications in the study countries [9]. In a recent review [32], health-related quality of life in older adults with multimorbidity was ranked as the most important research topic to improve their health and health care.

This study has several limitations. This was a cross-sectional study, so causal conclusions cannot be drawn. The investigation was conducted among chronic patients from conveniently selected health facilities in the study countries, and inclusion of other health facilities could have resulted in different findings. The preselection of chronic disease patients in this study design prevented us from reporting the overall prevalence of multimorbidity among out-patients, in general resulting in a probably higher prevalence of multimorbidity among chronic disease patients. A further limitation was that the rate of health utilization among the different chronic condition patients was not assessed. This study used a count of chronic conditions as a measure of multimorbidity, implying that each chronic condition has an equal impact on an individual, while in reality disease severity, the specific combination of chronic conditions, and access to health care may affect multimorbidity.

\section{Conclusions}

This study from public primary health care providers found a high chronic condition multimorbidity among chronic disease patients in four Greater Mekong countries. Common patterns of chronic condition multimorbidity-e.g., hypertension was strongly comorbid with depression, type 2 diabetes, CVD, and dyslipidaemia, and CVD was strongly comorbid with hypertension and depression-were identified that could help in the development of chronic condition multimorbidity guidelines and management plans in this setting. Older male patients of lower education in a rural area seem to be at the highest risk of multimorbidity, particularly in the presence of a poor diet.

Acknowledgments: This research project was supported by Mahidol University.

Author Contributions: Supa Pengpid and Karl Peltzer participated in the conception, design, and analysing data. Supa Pengpid wrote a draft of the paper, and all authors wrote and approved the final version of the manuscript.

Conflicts of Interest: The authors declare no conflict of interest. 


\section{References}

1. Low, W.Y.; Lee, Y.K.; Samy, A.L. Non-communicable diseases in the Asia-Pacific region: Prevalence, risk factors and community-based prevention. Int. J. Occup. Med. Environ. Health 2015, 28, 20-26. [CrossRef] [PubMed]

2. Narain, J.P.; Garg, R.; Fric, A. Non-communicable diseases in the South-East Asia region: Burden, strategies and opportunities. Natl. Med. J. India 2011, 24, 280-287. [PubMed]

3. Siegel, K.R.; Patel, S.A.; Ali, M.K. Non-communicable diseases in South Asia: Contemporary perspectives. Br. Med. Bull. 2014, 111, 31-44. [CrossRef] [PubMed]

4. World Health Organization (WHO). Noncommunicable Diseases in the South-East Asia Region: Situation and Response 2011; World Health Organization, Regional Office for South-East Asia: New Dehli, India, 2012.

5. Barnett, K.; Mercer, S.W.; Norbury, M.; Watt, G.; Wyke, S.; Guthrie, B. Epidemiology of multimorbidity and implications for health care, research, and medical education: A cross-sectional study. Lancet 2012, 380, $37-43$. [CrossRef]

6. Mercer, S.; Salisbury, C.; Fortin, M. ABC of Multimorbidity; John Wiley \& Sons: Hoboken, NJ, USA, 2014.

7. Xu, X.; Mishra, G.D.; Jones, M. Mapping the global research landscape and knowledge gaps on multimorbidity: A bibliometric study. J. Glob. Health 2017, 7, 010414. [CrossRef] [PubMed]

8. Violan, C.; Foguet-Boreu, Q.; Flores-Mateo, G.; Salisbury, C.; Blom, J.; Freitag, M.; Glynn, L.; Muth, C.; Valderas, J.M. Prevalence, determinants and patterns of multimorbidity in primary care: A systematic review of observational studies. PLoS ONE 2014, 9, e102149. [CrossRef] [PubMed]

9. Arokiasamy, P.; Uttamacharya, U.; Jain, K.; Biritwum, R.B.; Yawson, A.E.; Wu, F.; Guo, Y.; Maximova, T.; Espinoza, B.M.; Rodríguez, A.S.; et al. The impact of multimorbidity on adult physical and mental health in low- and middle-income countries: What does the study on global ageing and adult health (SAGE) reveal? BMC Med. 2015, 13, 178. [CrossRef] [PubMed]

10. Lalkhen, H.; Mash, R. Multimorbidity in non-communicable diseases in South African primary healthcare. S. Afr. Med. J. 2015, 105, 134-138. [CrossRef] [PubMed]

11. Sinnige, J.; Korevaar, J.C.; Westert, G.P.; Spreeuwenberg, P.; Schellevis, F.G.; Braspenning, J.C. Multimorbidity patterns in a primary care population aged 55 years and over. Fam. Pract. 2015, 32, 505-513. [CrossRef] [PubMed]

12. Ornstein, S.M.; Nietert, P.J.; Jenkins, R.G.; Litvin, C.B. The prevalence of chronic diseases and multimorbidity in primary care practice: A PPRNet report. J. Am. Board. Fam. Med. 2013, 26, 518-524. [CrossRef] [PubMed]

13. Phaswana-Mafuya, N.; Peltzer, K.; Chirinda, W.; Musekiwa, A.; Kose, Z.; Hoosain, E.; Davids, A.; Ramlagan, S. Self-reported prevalence of chronic non-communicable diseases and associated factors among older adults in South Africa. Glob. Health Action 2013, 6, 20936. [CrossRef] [PubMed]

14. Hussain, M.A.; Huxley, R.R.; Al Mamun, A. Multimorbidity prevalence and pattern in Indonesian adults: An exploratory study using national survey data. BMJ Open 2015, 5, e009810. [CrossRef] [PubMed]

15. Mini, G.K.; Thankappan, K.R. Pattern, correlates and implications of non-communicable disease multimorbidity among older adults in selected Indian states: A cross-sectional study. BMJ Open 2017, 7, e013529. [CrossRef] [PubMed]

16. Fortin, M.; Lapointe, L.; Hudon, C.; Vanasse, A.; Ntetu, A.L.; Maltais, D. Multimorbidity and quality of life in primary care: A systematic review. Health Qual. Life Outcomes 2004, 2, 51. [CrossRef] [PubMed]

17. Peltzer, K.; Pengpid, S.; Puckpinyo, A.; Yi, S.; Anh, L.V. The utilization of traditional, complementary and alternative medicine for non-communicable diseases and mental disorders in health care patients in Cambodia, Thailand and Vietnam. BMC Complement. Altern. Med. 2016, 16, 92. [CrossRef] [PubMed]

18. Peltzer, K.; Pengpid, S. Anticipated stigma in chronic illness patients in Cambodia, Myanmar and Vietnam. Nagoya J. Med. Sci. 2016, 78, 423-435. [CrossRef] [PubMed]

19. Lee, G.B.; Charn, T.C.; Chew, Z.H.; Ng, T.P. Complementary and alternative medicine use in patients with chronic diseases in primary care is associated with perceived quality of care and cultural beliefs. Fam. Pract. 2004, 21, 654-660. [CrossRef] [PubMed]

20. World Health Organization (WHO). Guidelines for Controlling and Monitoring the Tobacco Epidemic; WHO: Geneva, Switzerland, 1998. 
21. Bush, K.; Kivlahan, D.R.; McDonell, M.B.; Fihn, S.D.; Bradley, K.A. The AUDIT alcohol consumption questions (AUDIT-C): An effective brief screening test for problem drinking. Ambulatory Care Quality Improvement Project (ACQUIP). Alcohol Use Disorders Identification Test. Arch. Intern. Med. 1998, 158, 1789-1795. [CrossRef] [PubMed]

22. Zigmond, A.S.; Snaith, R.P. The hospital anxiety and depression scale. Acta Psychiatr. Scand. 1983, 67, 361-370. [CrossRef] [PubMed]

23. Gaskins, N.; Sloane, P.; Mitchell, C.; Ammerman, A.; Ickes, S.; Williams, C. Poor nutritional habits: A modifiable predecessor of chronic illness? A North Carolina Family Medicine Research Network (NC-FM-RN) study. J. Am. Board Fam. Med. 2007, 20, 124-134. [CrossRef] [PubMed]

24. World Health Organisation (WHO). Global Physical Activity Surveillance. 2009. Available online: http: / / www.who.int/chp/steps/GPAQ/en/index.html (accessed on 2 December 2016).

25. Schmidt, S.; Mühlan, H.; Power, M. The EUROHIS-QOL 8-item index: Psychometric results of a cross-cultural field study. Eur. J. Public Health 2006, 16, 420-428. [CrossRef] [PubMed]

26. Da Rocha, N.S.; Power, M.J.; Bushnell, D.M.; Fleck, M.P. The EUROHIS-QOL 8-item index: Comparative psychometric properties to its parent WHOQOL-BREF. Value Health 2012, 15, 449-457. [CrossRef] [PubMed]

27. Clarke, D.M.; Currie, K.C. Depression, anxiety and their relationship with chronic diseases: A review of the epidemiology, risk and treatment evidence. Med. J. Aust. 2009, 190, S54-S60. [PubMed]

28. Mendenhall, E.; Norris, S.A.; Shidhaye, R.; Prabhakaran, D. Depression and type 2 diabetes in low- and middle-income countries: A systematic review. Diabetes Res. Clin. Pract. 2014, 103, 276-285. [CrossRef] [PubMed]

29. Yohannes, A.M.; Willgoss, T.G.; Baldwin, R.C.; Connolly, M.J. Depression and anxiety in chronic heart failure and chronic obstructive pulmonary disease: Prevalence, relevance, clinical implications and management principles. Int. J. Geriatr. Psychiatry 2010, 25, 1209-1221. [CrossRef] [PubMed]

30. Sinnige, J.; Braspenning, J.; Schellevis, F.; Stirbu-Wagner, I.; Westert, G.; Korevaar, J. The prevalence of disease clusters in older adults with multiple chronic diseases-A systematic literature review. PLoS ONE 2013, 8, e79641. [CrossRef] [PubMed]

31. Van Weel, C.; Schellevis, F.G. Comorbidity and guidelines: Conflicting interests. Lancet 2006, 367, 550-551. [CrossRef]

32. Tisminetzky, M.; Bayliss, E.A.; Magaziner, J.S.; Allore, H.G.; Anzuoni, K.; Boyd, C.M.; Gill, T.M.; Go, A.S.; Greenspan, S.L.; Hanson, L.R.; et al. Research priorities to advance the health and health care of older adults with multiple chronic conditions. J. Am. Geriatr. Soc. 2017, 65, 1549-1553. [CrossRef] [PubMed] 